\title{
Modelling line edge roughness in periodic line-space structures by Fourier optics to improve scatterometry
}

\section{H. Gross}

hermann.gross@ptb.de

\section{S. Heidenreich}

\author{
M-A. Henn \\ G. Dai
}

F. Scholze

M. Bär

\author{
Department Mathematical Modelling and Data Analysis, Physikalisch-Technische Bundesanstalt \\ (PTB), Berlin, Germany \\ Department Mathematical Modelling and Data Analysis, Physikalisch-Technische Bundesanstalt \\ (PTB), Berlin, Germany \\ Department Mathematical Modelling and Data Analysis, Physikalisch-Technische Bundesanstalt \\ (PTB), Berlin, Germany \\ Department Dimensional Nanometrology, Physikalisch-Technische Bundesanstalt (PTB), Braun- \\ schweig, Germany \\ Department Radiometry with Synchrotron Radiation, Physikalisch-Technische Bundesanstalt (PTB), \\ Berlin, Germany \\ Department Mathematical Modelling and Data Analysis, Physikalisch-Technische Bundesanstalt \\ (PTB), Berlin, Germany
}

In the present paper, we propose a 2D-Fourier transform method as a simple and efficient algorithm for stochastical and numerical studies to investigate the systematic impacts of line edge roughness on light diffraction pattern of periodic line-space structures. The key concept is the generation of ensembles of rough apertures composed of many slits, to calculate the irradiance of the illuminated rough apertures far away from the aperture plane, and a comparison of their light intensities to those of the undisturbed, 'non-rough' aperture. We apply the Fraunhofer approximation and interpret the rough apertures as binary $2 \mathrm{D}$-gratings to compute their diffraction patterns very efficiently as the $2 \mathrm{D}$-Fourier transform of the light distribution of the source plane. The rough edges of the aperture slits are generated by means of power spectrum density (PSD) functions, which are often used in metrology of rough geometries. The mean efficiencies of the rough apertures reveal a systematic exponential decrease for higher diffraction orders if compared to the diffraction pattern of the unperturbed aperture. This confirms former results, obtained by rigorous calculations with computational expensive finite element methods (FEM) for a simplified roughness model. The implicated model extension for scatterometry by an exponential damping factor for the calculated efficiencies allows to determine the standard deviation $\sigma_{\mathrm{r}}$ of line edge roughness along with the critical dimensions (CDs), i.e., line widths, heights and other profile properties in the sub-micrometer range. First comparisons with the corresponding roughness value determined by $3 \mathrm{D}$ atomic force microscopy (3D AFM) reveal encouraging results.

[DOI: http://dx.doi.org/10.2971/jeos.2014.14003]

Keywords: Scatterometry, line edge roughness, power spectrum density, atomic force microscopy

\section{INTRODUCTION}

Scatterometry is widely used to evaluate precision of diffractive elements in lithography [1]. Extreme ultraviolet (EUV) scatterometry using light with wavelengths in a small range around $13.5 \mathrm{~nm}$ is an adequate tool for the characterization of photo masks and wafers [2]-[4]. The critical dimensions (CDs) of such elements decrease continuously with the progress in technology. A detailed description of the complex architecture of a typical EUV mask can be found in [5]. Many diffraction orders can be measured by EUV scatterometry, with the wavelengths being distinctly shorter than the typical dimensions of the structures to be investigated. The diffraction orders measured are typically in the range of \pm 10 where their efficiencies are larger than the disturbing background noise. This provides information on the higher frequency range corresponding to the spatial frequency resolution of the profile functions describing the geometry, i.e., informations on the tiny details of the structure. Obviously, the structure roughness with am- plitudes in the range of a few nanometers has an impact too, and can no longer be neglected in the course of the profile reconstruction.

In previous publications a comparison of the reconstructed profiles using EUV-scatterometry and the results obtained using atomic force and electron microscopy has revealed that scatterometry underestimates the side-wall angle by several degrees. Imperfect modelling is supposed to be one of the main reasons for this [6]-[8]. In particular, to get reliable simulations and reconstructions, line edge roughness (LER) has to be taken into account. Recently, Kato and Scholze [8] have suggested approximative analytical expressions for the systematic corrections of the scattered efficiencies stemming from LER. They have applied Fraunhofer's diffraction method, i.e., the Fourier transform of the reflectivity function of a perturbed binary grating. They found damping of the mean ef- 
ficiencies with increasing diffraction orders, which was confirmed by rigorous FEM simulations for a real EUV mask [9]. In these FEM investigations, large computational domains containing many line-space structures with stochastically chosen widths were used. The roughness model used in [9] is strictly speaking one-dimensional, which inherently only describes in-plane scattering between the diffraction orders. The center positions and the widths of lines within the cross section of an EUV grating containing several periods are randomly changed. That means, that the line edges of this roughness model are still straight lines and no autocorrelation function along the edges is used, i.e., a crude LER model is applied. The calculations themselves are based on a rigorous solution of the 2D Helmholtz equation by the finite element method. If the geometry and material properties are invariant in one direction, here in the direction perpendicular to the plane of the cross section, Maxwell equations can be reduced to the 2D Helmholtz equation.

These results were still obtained for a simplified model of rough line edges, i.e., without means of an autocorrelation function along the edges, which is often used in metrology of rough geometries. There are several publications [10]-[12], where the modelling of line edge roughness as a stochastic process starts with an exponentially decaying autocorrelation function for the position $p(r)$ of an edge point at distance $r$ : $p(r)=\sigma^{2} e^{-(r / \xi)^{2 \alpha}}$ where $\sigma$ is the standard deviation, $\xi$ is the linear correlation length, and $\alpha$ is a roughness exponent (also referred to as the Hurst exponent). Randomised line edge profiles can be generated by calculating or approximating the associated power spectrum density function $\operatorname{PSD}\left(r^{-1}\right)$ belonging to the autocorrelation function $p(r)$ and subsequently applying an inverse Fourier transform with a random phase uniformly distributed in the range of $[0,2 \pi]$. For instance, Bergner et al. [10] use a similar approach to generate rough line edge profiles of 2D-binary gratings to calculate its impact on the angular dependence of the specular, 0th order reflectance at wavelengths around $633 \mathrm{~nm}$. Torcal-Milla et al. [13] have investigated gratings with rough edges in the visible optical range by applying the Rayleigh-Sommerfeld approach for near field simulations and the Fraunhofer approximation for the far field pattern. They found an exponential attenuation of the light intensities in terms of $\sigma$ and the diffraction order as well. The values for $\sigma$ they applied were in the range of several $\mu \mathrm{m}$ and they used a simplified statistical approach without considering a roughness exponent $\alpha$. Schuster et al. [14] have studied the impact of LER for silicon gratings on the basis of sinusoidal perturbations for the line positions with amplitudes in the range of $2-8 \mathrm{~nm}$ and for wave-lengths of 400 and $250 \mathrm{~nm}$, respectively.

The main issue of the presented investigations is to clarify the impact of the aperiodic perturbations due to different patterns of line roughness in line-space structures on scattering efficiencies of higher diffraction orders. The efficiencies are measured in the far field and in a plane perpendicular to the lines. Assuming a linear and isotropic dielectric medium where additionally the material properties are invariant in one direction, e.g. along the line-space structures, the electric field $\mathrm{E}$ and the magnetic field $\mathrm{H}$ are decoupled and scalar diffraction equations can be applied. Under these conditions the Fraun- hofer far-field approximation is applied to a $2 \mathrm{D}$ source field distribution $U_{1}(\xi, \eta)$ (cf. Figure 3 ) which is characterized by a binary field distribution. This can be interpreted as a thin-film model of the geometry neglecting any layer structures along the $z$ direction perpendicular to the source plane. In this sense the proposed model of LER is focused on perturbations of the periodicity of the line-space structures and their impact on the efficiencies. It is shown that this impact of line roughness of $2 \mathrm{D}$ structures is given by an exponential damping factor applied to the undisturbed efficiencies of the non-rough structures. This remains valid for different kinds of roughness patterns. The method can be applied to samples with different line to space ratios including high aspect ratios. For the samples presented here, EUV scatterometry with wavelengths $\lambda$ in the range of $13.5 \mathrm{~nm}$ and structures of about $100 \mathrm{~nm}$ critical dimensions, the existence of many orders of diffraction is given and the feasibility of the approximation is demonstrated. Even for smaller critical dimensions, e.g. $30 \mathrm{~nm}$, several diffraction orders are available and the bias of the mean efficiencies can be determined according to proposed method. But for line-space structures with periods smaller than the used wavelength no other diffraction orders aside from the zeroth order exist and other methods such as the effective layer model [10] must be used to consider roughness.

To resolve the LER-induced correction for different roughness patterns a randomization based on PSD functions describing the roughness pattern is applied. We will restrict our investigations to diffraction orders in the range of \pm 10 whose efficiencies are significantly greater than the background noise of the measurement setup, i.e., larger than $0.001 \%$ if the efficiencies are given in per cent. The model approximation errors are significantly smaller than the $0.001 \%$. They are dependent on the precision of the 2D Fourier transform procedure and the discretization of the source plane. The line edge roughness pattern is controlled by an autocorrelation function depending on a standard deviation $\sigma$, a correlation length $\xi$ and a roughness exponent $\alpha$. The key concept is to generate ensembles of apertures composed of rough slits, to calculate the irradiance of the illuminated rough apertures far away from the aperture plane, and to compare their light intensities to those of the unperturbed, 'non-rough' aperture. In order to avoid computational expensive FEM calculations, we compute the diffraction pattern very efficiently as the 2D-Fourier transform of the light distribution of a binary source plane applying the Fraunhofer approximation.

The proposed method is very sensitive to the imposed line edge roughness. We have analyzed ensembles of rough apertures with varying values for the standard deviation, the correlation length and the roughness exponent. The standard deviation $\sigma$ of the imposed LER has an essential effect on the bias of the mean efficiencies. Relative to their unperturbed values stemming from 'non-rough' apertures, the light intensities of higher diffraction orders $n_{j}$ are damped by $\exp \left(-\frac{4 \pi^{2}}{d^{2}} n_{j}^{2} \sigma^{2}\right)$, where $d$ is the period of the slits in the aperture. Interpreting the rough apertures as binary 2D-gratings, the reported former findings [9], obtained by expensive FEM calculations for a relative crude LER model, are approved. That emphasizes to include the exponential damping factor in scatterometric modelling to get more reliable results for the reconstructed 
CDs. Furthermore, the model extension allows to determine the roughness parameter $\sigma$ along with the geometrical profile parameters. Note that such a determination of the $\sigma$ value for the standard deviation of randomised line edge roughness is based on an integral measurement principle. We indicate this reconstructed roughness parameter as $\sigma_{\mathrm{r}}$. For the measurements reported here, the typical beam size at the sample during an EUV measurement is about $1 \mathrm{~mm}$ in both directions and several thousand lines are interacting with the incidental light.

This paper is organized as follows: In Section 2 a brief description of how the ensembles of rough apertures were generated on the basis of a stochastic process is given. Furthermore a short derivation of the formula for the light intensities far away from the aperture applying the Fraunhofer approximation is discussed. Section 3 contains the results obtained for different ensembles of rough binary 2D-gratings and rough apertures, respectively. With apertures typically composed of four slits, a size of $1 \mu \mathrm{m}$ in both directions, and a pixel size of $0.1 \mathrm{~nm}$ in $\mathrm{x}$ - and $\mathrm{y}$-direction we get a sufficient sensitivity for the calculated far-field irradiance of the corresponding diffraction orders in the range \pm 10 . It is shown, that the spatial averaging for each sample of a rough aperture is so strong, that just a few ten samples, e.g., 30, are sufficient to get a stable bias for the mean values at the different diffraction orders. In Section 4 scatterometric reconstructions of the CDs of an EUV photo mask based on rigorous FEM calculations and maximum likelihood estimation are reinvented $[15,16]$ to demonstrate the improvements and the feasibility to determine $\sigma_{\mathrm{r}}$ simultaneously. First comparisons with the results of 3D atomic force microscopy (3D AFM) measurements on the same EUV mask endorse the investigations. Section 5 closes the paper with a discussion of the results and the conclusions.

\section{ENSEMBLES OF ROUGH 2D LINE-SPACE STRUCTURES}

\subsection{Apertures with rough edges}

Representing 2D-binary gratings, we are creating square arrays of strip shaped slits, i.e., square apertures composed of many slits whose distribution of reflectivity equals 0 or 1 , depending on the position in the plane of the aperture. In order to get apertures with rough boundary lines, the variations along the line edges are derived by an exponentially decaying autocorrelation function. Considering a line along the $\mathrm{y}$ direction $\{(x(y), y): y \in R\}$ with random variables $x(y)$, we assume a constant mean value $\langle x(y)\rangle=x_{0}$ and that the correlation

$$
x\left(y_{1}, y_{2}\right):=\frac{\left\langle\left[x\left(y_{1}\right)-x_{0}\right]\left[x\left(y_{2}\right)-x_{0}\right]\right\rangle}{x_{0}^{2}}
$$

depends on the distance $r=\left|y_{1}-y_{2}\right|$ only, i.e. $x\left(y_{1}, y_{2}\right)=x(r)$. Moreover, we presume the following autocorrelation function

$$
x(r)=\sigma^{2} e^{-(r / \xi)^{2 \alpha}},
$$

where $\sigma$ is the standard deviation of the edge positions, $\xi$ is the linear correlation length along the line, and $\alpha$ is the
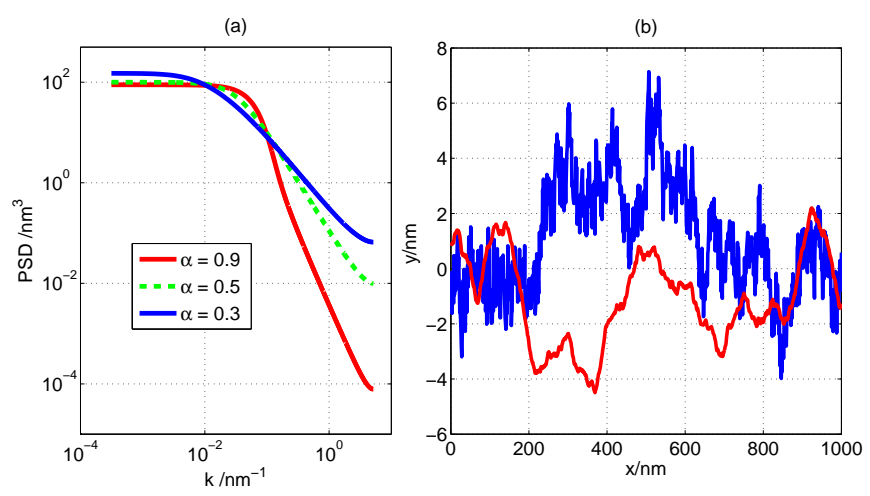

FIG. 1 Power spectral density (PSD) and generated rough lines a) for $\sigma=1 \mathrm{~nm}$, correlation length $\xi=50 \mathrm{~nm}$ and three different roughness exponents $\alpha=0.3,0.5,0.9$; (b) samples of rough lines calculated by the corresponding PSD with $\alpha=0.3$ and 0.9 .

so-called roughness exponent. Recent publications, e.g. Mack and Bergner et al. [10]-[12], are starting with such an exponentially decaying function for modelling a stochastic process which ends up in randomised line edges or surfaces. Our investigations are addressing the 1D case of randomly roughened lines. Randomised line edge profiles are generated by calculating or approximating the associated power spectrum density function $P S D\left(r^{-1}\right)$ that belongs to the autocorrelation function $p(r)$ and subsequently applying an inverse Fourier transform with a random phase being uniformly distributed in the range of $[0,2 \pi]$. By this means the generated rough line edges shown in Figure 1 were generated for different roughness exponents $(\alpha=0.3$ and $\alpha=0.9)$ and for a standard deviation $\sigma=1 \mathrm{~nm}$ and a linear correlation length $\xi=50 \mathrm{~nm}$. It is revealed that higher values of $\alpha$ lead to reduced higher frequency components in the variances of the edges, i.e., they become significantly smoother. The edges of the aperture slits are constituted by repeating the creation process independently from each other. Bergner et al. [10] use a similar approach to generate rough line edge profiles of 2D-binary gratings to calculate its impact on the angular dependence of the specular, 0th order reflectance at wavelengths around $633 \mathrm{~nm}$.

To get a sufficient resolution, the pixel size is $0.1 \mathrm{~nm}$ in both directions of the $2 \mathrm{D}$ apertures broadening upon a total range of $1 \mu \mathrm{m} \times 1 \mu \mathrm{m}$. The investigated rough apertures are typically composed of four periodically arranged slits, i.e., the corresponding grating period is $250 \mathrm{~nm}$ and the mean width of the rough slits is $125 \mathrm{~nm}$. Figure 2 show two examples illustrating the impact of higher values of $\alpha$ and $\xi$, respectively. There is no restriction to choose other line-to-space ratios (cf. Figure 4(a)). We choose several slits per apertures to improve the spatial averaging of the irradiance pattern associated with the randomised aperture.

\subsection{Fraunhofer approximation}

Fourier optics is well known for being a cornerstone for the analysis of imaging, diffraction, coherence and propagation through random media $[17,18]$. The mathematical description of the propagation of an optical field from one location, for example an diffractive aperture, to another is one of its 

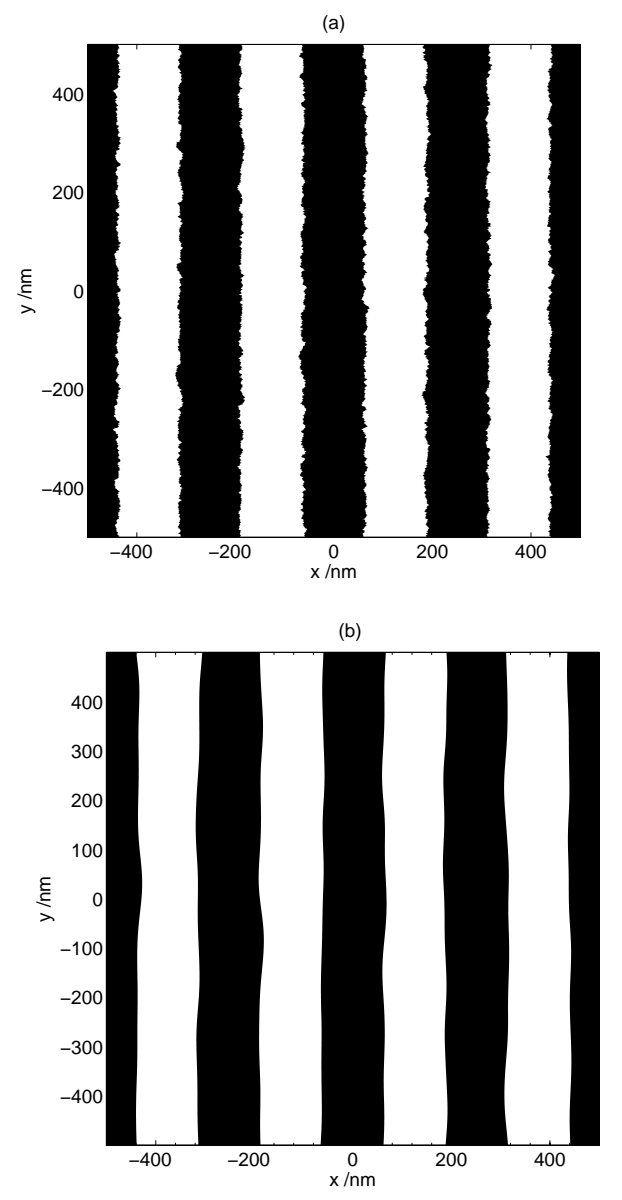

FIG. 2 Two examples of a rough apertures; size $1 \mu \mathrm{m}$ in both directions with a resolution of $0.1 \mathrm{~nm}$ ( $\Longleftrightarrow 10000 \times 10000$ points); four slits per aperture, i.e., $125 \mathrm{~nm}$ width of the slits and a period of $250 \mathrm{~nm}$; (a) used PSD with $\sigma=3 \mathrm{~nm}, \alpha=0.5$, and $\xi=10 \mathrm{~nm}$ and (b) with $\sigma=3 \mathrm{~nm}, \alpha=1.0$, and $\xi=100 \mathrm{~nm}$.

most essential tasks. In general the propagation behavior of electro- magnetic waves in matter is based on Maxwell's equations, where the electric field $E$ and the magnetic field $H$ are coupled. However, assuming a linear and isotropic dielectric medium where additionally the material properties are invariant in one direction, both fields are decoupled and scalar diffraction equations can be applied.

Under these ideal conditions a monochromatic plane wave with wavenumber $k$ and orthogonal incidence on a diffractive aperture is considered. Its propagating radiation in an parallel observation plane far away from the aperture can be elegantly expressed as the Fourier transform of the field distribution in the source plane applying the Fraunhofer approximation. According to Huygens' principle each point of the wave front in the source plane can be considered as the source of superimposing spherical waves generating secondary wave fronts. That is, the field distribution $U_{2}(x, y)$ in an observation plane parallel to the source plane (cf. Figure 3 ) can be calculated by means of the field distribution $U_{1}(\xi, \eta)$ of the source plane:

$$
U_{2}(x, y)=\frac{z}{j \lambda} \iint_{\Omega} U_{1}(\xi, \eta) \frac{e^{j k r}}{r^{2}} d \xi d \eta
$$

here given in Rayleigh-Sommerfeld's diffraction notation with

$$
r=\sqrt{z^{2}+(x-\xi)^{2}+(y-\eta)^{2}}
$$

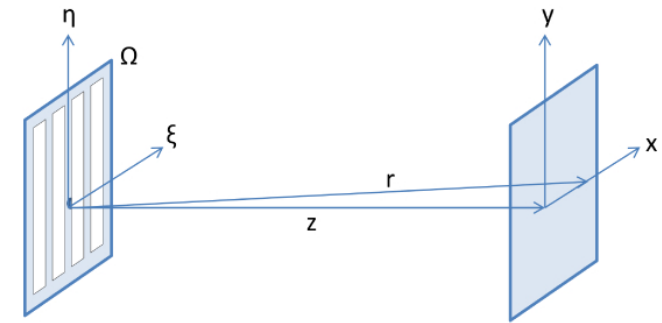

source plane with given $U_{1}(\xi, \eta)$

observation plane with sought $\mathrm{U}_{2}(\mathrm{x}, \mathrm{y})$

FIG. 3 Scheme of the coordinate system used in the calculations.

and $\Omega$ as the domain of the source plane. It can be shown very easily (cf. $[19,20])$ that for propagation distances that are very long compared to the size of the aperture, i.e., for distances $z$ with

$$
z \gg \max _{(\xi, \eta) \in \Omega} \frac{k\left(\xi^{2}+\eta^{2}\right)}{2},
$$

$U_{2}(x, y)$ from Eq. (2) can be written as

$$
U_{2}(x, y)=\frac{e^{j k z}}{j \lambda z} e^{\frac{j k\left(x^{2}+y^{2}\right)}{2 z}} \iint_{\Omega} U_{1}(\xi, \eta) e^{\frac{-j 2 \pi}{\lambda z}(x \xi+y \eta)} d \xi d \eta .
$$

Eq. (5) represents the Fraunhofer diffraction expression. It consists of a complex exponential function times the Fourier transform of the source field $U_{1}$ with the following frequency variable substitutions

$$
f_{\xi} \rightarrow \frac{x}{\lambda z}, f_{\eta} \rightarrow \frac{y}{\lambda z}
$$

Note that the multiplicative complex exponentials in front of the double integral disappear, provided only the irradiance is of interest. This is usually the case when calculating the Fraunhofer pattern of an aperture.

We apply this Fraunhofer expression to the calculation of the irradiance pattern of the rough apertures and the corresponding unperturbed non-rough aperture for a wavelength of $\lambda$ $=13.5 \mathrm{~nm}$ and a distance $z=1 \mathrm{~m}$ of the observation plane. Figure 4 shows an example for an aperture composed of four periodically arranged slits with a width of $200 \mathrm{~nm}$ and a period of $250 \mathrm{~nm}$, i.e., the line-to-space ratio is 1:4. The irradiance pattern along the $x$-direction at the central position $y=0$ is shown for the unperturbed aperture. The shape of the intensity distribution along the diffraction orders is typical for the chosen line-to-space ratio and an incident angle of $0^{\circ}$ (cf. [6]). Note that depending on the bridge-slit ratio equivalent to the line-space ratio of $2 \mathrm{D}$ binary grating, the irradiance becomes zero for special diffraction orders. These diffraction orders are rejected for the evaluations and only those with values significantly greater than zero are selected. In the example of Figure 4 this is the case for the orders $n_{j}= \pm 5$.

\section{IMPACT OF DIFFERENT PATTERNS OF LINE EDGE ROUGHNESS}

\subsection{Irradiance pattern relative to those of 'non-rough' structures}

The key for analysing the impact of roughness is to compare the mean light intensities calculated for ensembles of rough 

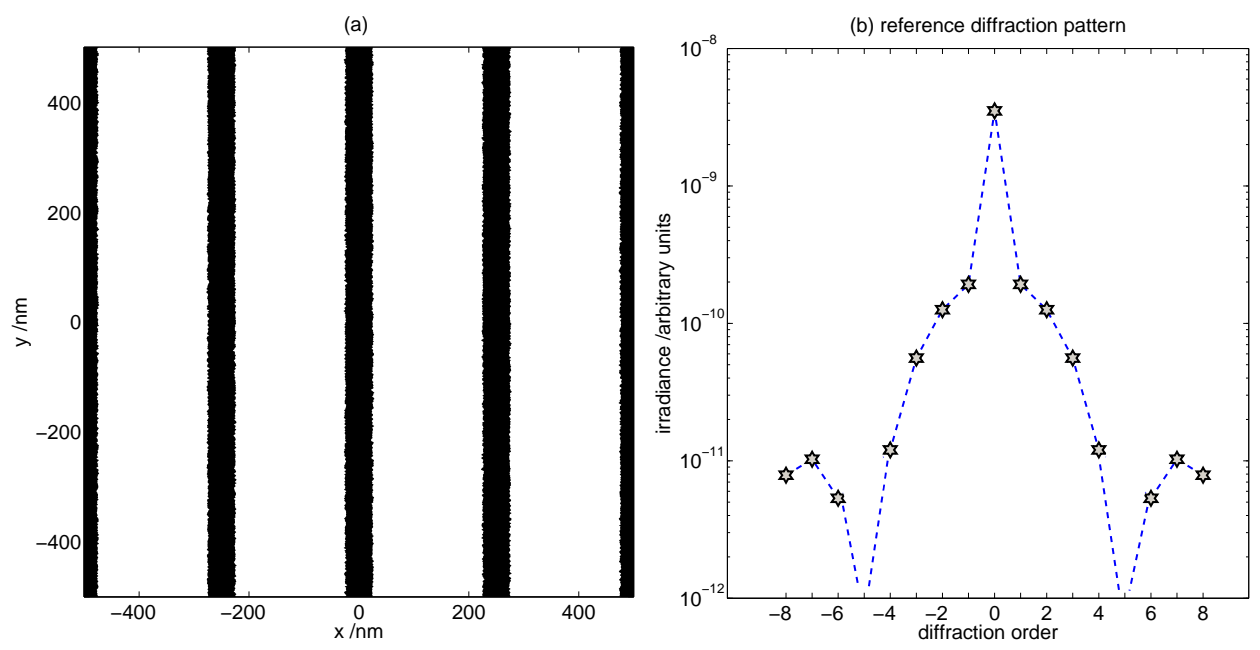

FIG. 4 (a) Rough aperture $(\sigma=1 \mathrm{~nm} ; \alpha=1 ; \xi=2 \mathrm{~nm}$ ) with a slit width of $200 \mathrm{~nm}$ at a period of $250 \mathrm{~nm}$ and (b) reference irradiance pattern of the corresponding unperturbed aperture (with straight edges).

apertures to that of the unperturbed non-rough aperture, i.e., the corresponding aperture whose slits are composed of straight lines. Any systematic impact on the mean efficiencies should then be identified obviously. In previous investigations that made use of computational expensive FEM simulations for a 2D computational domain [9], this strategy has already been applied successfully. Note that the applied roughness model of these studies was very simple, i.e., only the center locations and widths of neighboring lines were stochastically chosen and unfortunately no perturbations along the line direction could be treated in the former, cross section oriented FEM approach.

The relative deviations of light intensities in dependence of the diffraction orders for several ensembles of rough apertures are depicted in Figure 5. The three examples differ from each other by an increasing standard deviation $\sigma$ of the autocorrelation function (cf. Eq. (1)) from $2 \mathrm{~nm}$ to $5 \mathrm{~nm}$ used to generate the rough edges of the apertures. The roughness exponent $\alpha$ was fixed to 1.0 and the linear correlation length $\xi$ was set to $10 \mathrm{~nm}$. Each ensemble has elven samples of rough apertures whose relative deviations are depicted as circle symbols. Their mean values are marked by diamond symbols. The bridge to slit ratio was $1: 1$, i.e., both parameters have a nominal value of $125 \mathrm{~nm}$. Again only the orders with intensities greater than zero are considered.

A systematic nonlinear decrease of the mean efficiencies for higher diffraction orders along with slightly increasing variances is observed. This is established for different degrees of roughness expressed by the different $\sigma$ values for three ensembles. Only a few ten samples for each ensemble are necessary to reveal a stable bias of the mean efficiencies.

Quite similar outcomes are obtained for the three ensembles generated with different correlation lengths $\xi=\{10,60,120\}$ $\mathrm{nm}$ as shown in Figure 6. Here $\alpha$ was changed to 0.5 and $\sigma$ was fixed to $3 \mathrm{~nm}$. Obviously, the correlation length $\xi$ has only an effect on the variances around the mean values of the light intensities. They rise slightly in particular for higher diffraction orders. Nevertheless, the essential impact on the biased mean values is stemming from the imposed $\sigma$ values and appears to follow an exponential function depending on the diffraction order and $\sigma$, just like the above-mentioned findings of the 2D FEM simulations.

Consequently, we conclude that the revealed attenuation of the mean light intensities with higher diffraction orders can be modelled by

$$
\begin{aligned}
& \frac{f_{n_{j}, \text { ref }}-\overline{f_{n_{j}, \text { pert }}}}{f_{n_{j}, \text { ref }}} \approx 1-e^{-\left(\frac{2 \pi n_{j}}{d}\right)^{2} \sigma_{\mathrm{r}}^{2}} \\
\Longleftrightarrow & \overline{f_{n_{j}, \text { pert }}} \approx e^{-\left(\frac{2 \pi n_{j}}{d}\right)^{2} \sigma_{\mathrm{r}}^{2}} f_{n_{j}, \text { ref } .}
\end{aligned}
$$

Here $f_{n_{j} \text {,ref }}$ denotes the light intensities of the unperturbed aperture at diffraction order $n_{j}$ and $\overline{f_{n_{j} \text {,pert }}}$ the corresponding mean values of the generated ensemble of rough apertures, $d$ is the period of the bridge-slit structure and $\sigma_{\mathrm{r}}$ depicts that value for the standard deviation which represents the best-fit results for the mean normalized deviations applying Eq. (7). In fact, the solid lines in Figures 5 and 6 are depicting these best-fit results for $\sigma_{\mathrm{r}}$ obtained by minimizing the norm $\left\|f_{n_{j}, \text { pert }}-e^{-\left(\frac{2 \pi n_{j}}{d}\right)^{2} \sigma_{\mathrm{r}}^{2}} f_{n_{j} \text {,ref }}\right\|$. Note that this means that the evaluated $\sigma_{\mathrm{r}}$ values are good estimations of the imposed standard deviations used for the generation of the ensembles of rough apertures.

Figure 7 summarizes these findings for many different samples of rough apertures representing 2D-binary gratings. For three different values of $\sigma=[1,2,3] \mathrm{nm}$ and nine values of the correlation length along the edges $\xi=[5,10,20,40,60,80,100$, $120,150] \mathrm{nm}$, the bias of the mean efficiencies expressed by $\sigma_{\mathrm{r}}$ according to Eq. (7) has been evaluated. The roughness exponent $\alpha$ was set to 1.0 and 0.5 . Only a slight increase within a range of maximal $5 \%$ is found for the determined $\sigma_{\mathrm{r}}$ compared to the imposed standard deviation $\sigma$ of the associated rough ensemble. Hence, the influence of different correlation lengths $\xi$ and roughness exponents $\alpha$ on our hypothesis (7) is fairly weak. 

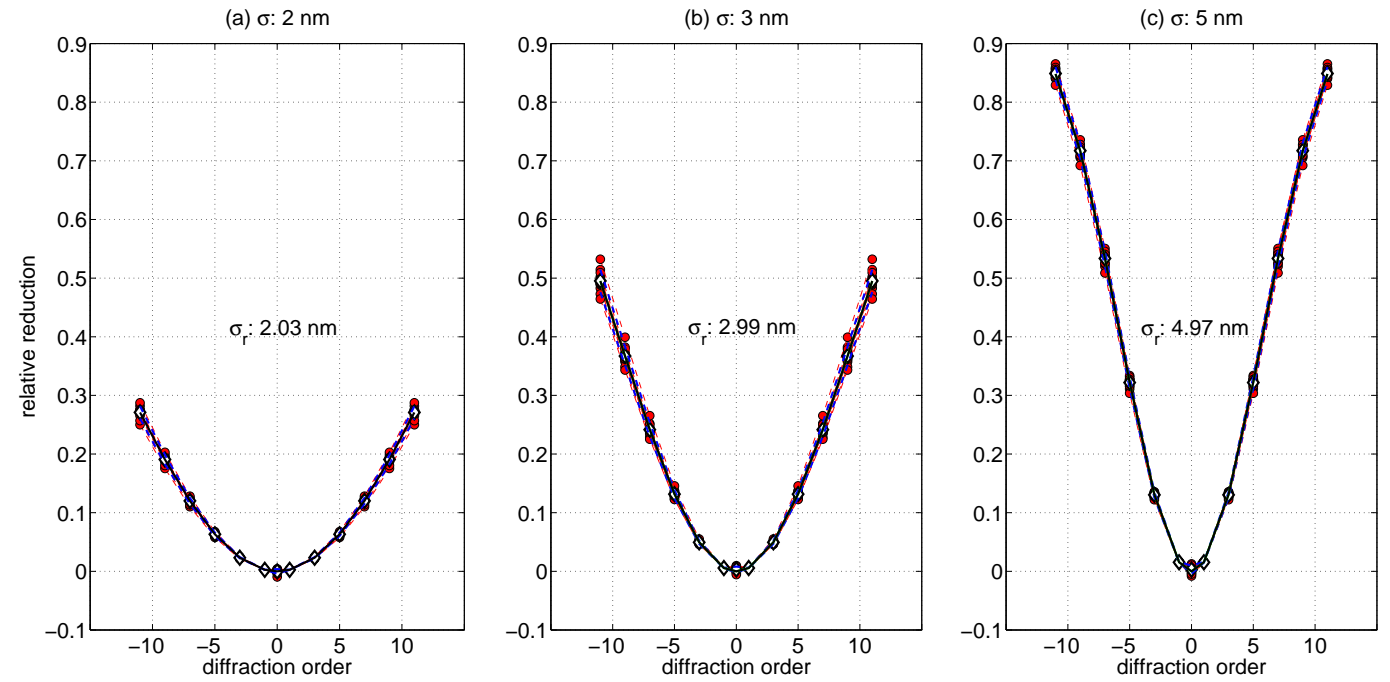

FIG. 5 Normalized deviations from the efficiencies of the unperturbed aperture, depicted as circles; diamond symbols represent the mean deviations of all samples and solid line the exponential approximation according Eq. (7); (a)-(c) for three different standard deviations $\sigma$ and $\alpha=1, \xi=10 \mathrm{~nm}$.
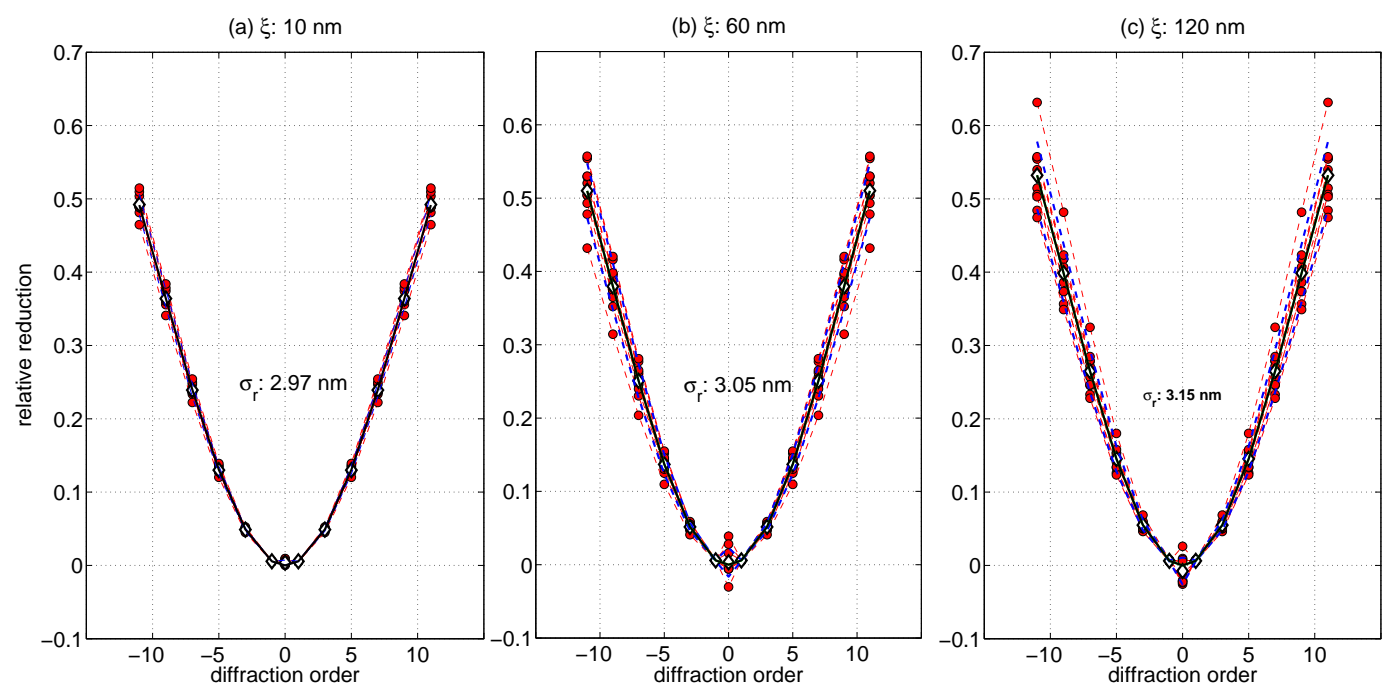

FIC. 6 Normalized deviations from the efficiencies of the unperturbed aperture, depicted as circles; diamond symbols represent the mean deviations of all samples and solid line the exponential approximation according Eq. (7); (a)-(c) for three different linear correlation lengths $\xi$ and $\sigma=3 \mathrm{~nm}, \alpha=0.5$.

\subsection{Comparison to previous 2D FEM approach}

Finally, we do compare the results directly with the normalized deviations provided by the above-mentioned rigorous 2D-FEM simulations over randomly perturbed cross sections of an EUV photo mask composed of different layers [9]. The chosen example has been calculated with 24 lines per computational domain overspreading the profile cross section with a randomised distribution of line and space widths of the neighbouring lines (cf. Fig. 3 a in [9]). The results shown in Figure 8 have been obtained by adapting the line and space widths in the 2D Fourier transform method to the corresponding values, i.e., to $93.33 \mathrm{~nm}$ for the lines and $186.67 \mathrm{~nm}$ for the space and choosing $\alpha=0.5$ and $\xi=10 \mathrm{~nm}$. The imposed $\sigma$ value for the edge roughness was $5.6 \mathrm{~nm}$ for both cases. The mean normalized deviations and the evaluated $\sigma_{\mathrm{r}}$ values of $5.49 \mathrm{~nm}$ and $5.59 \mathrm{~nm}$ for the exponentially damped efficiencies show a good agreement. Note that for the rigorous FEM approach 1000 samples per ensemble were applied, whereas for the Fourier approach only 32 samples were calculated to get the depicted stable bias with relatively small variances. Different values of $\alpha$ and $\xi$ have no significant impact on the comparison, i.e., good agreements are revealed as well. On a Linux workstation with 12 Intel Xeon processors (X5460@3.16 $\mathrm{GHz}$ ) the computation for one FEM simulation of a cross section containing 24 lines with randomly changed center positions and widths takes about 15 min. Hence, on a high performance cluster the computation of 1000 diffraction patterns representing the roughness example of Figure 8(a) needs typically 1-2 days depending on the available cluster resources. The corresponding 2D-Fourier calculations of an ensemble of rough apertures, such as that in Figure 8(b), need only a quarter of an hour on the linux workstation.

Probably the application of Fourier modal method or finitedifference time-domain (FDTD) analysis would required less computation time compared to the finite element method ap- 
plied in [9], but the comparison is not part of the present paper and could be addressed in further investigations. In principle a three-dimensional simulation of line edge roughness based on an autocorrelation function and realized for example by FEM is possible, but requires a tremendous amount of memory considering the required volume discretization (at least $\leq \lambda / 4$ for each direction), the necessary size of the volume (for example $0.2^{3} \mu \mathrm{m}^{3}$ ) and the size of roughness amplitudes in the one nanometer range.

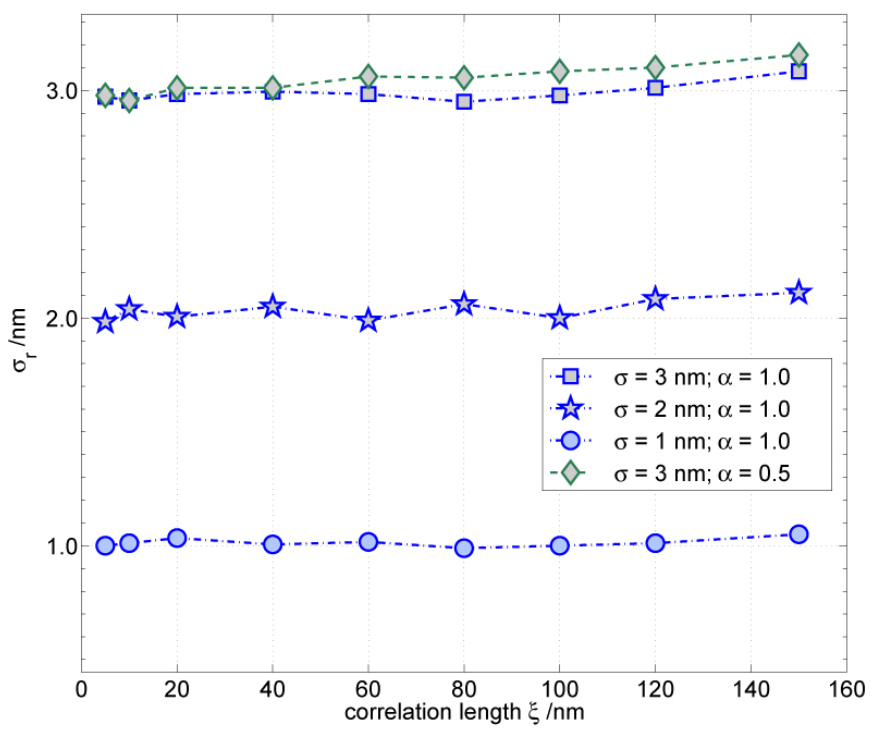

FIG. 7 Reconstructed values for $\sigma_{\mathrm{r}}$ for ensembles of rough apertures (2D-binary gratings) generated with standard deviations $\sigma$, linear correlation lengths $\xi$ and roughness exponents $\alpha$; Eq. (7) was applied to fit the mean biased efficiencies.

(a) $\sigma=5.6 \mathrm{~nm} ; 1000$ samples

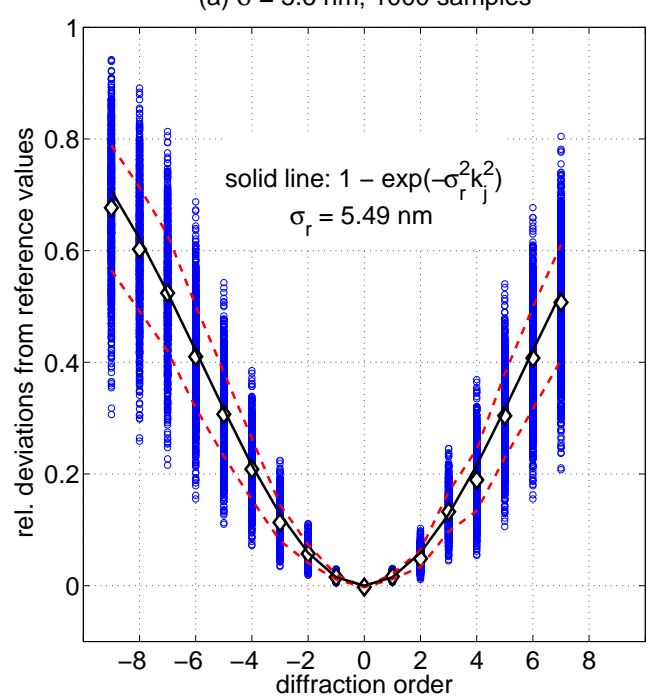

\section{ROUGHNESS AMPLITUDES DETERMINED BY SCATTEROMETRY AND AFM}

\subsection{Scatterometric model supplemented with line roughness}

The determination of the CDs from measured light diffraction pattern for a real photo mask is a challenging task requiring a rigorous calculation of the diffracting light efficiencies, e.g. by the finite element method. Solving the inverse problem amounts to determining the geometry of an optical grating whose light diffraction pattern fits a given set of measurement data best. Like many inverse problems, the inverse problem of scatterometry is ill-posed [21] and its treatment requires a priori information. A common approach for its regularization is to set up an equivalent low dimensional optimisation problem with a weighted objective function that is minimized using iterative algorithms $[6,22]$. The a priori information in this case not only includes information about the geometrical profile of the investigated element, but also knowledge of the variances of the measured data in order to describe the weighting factors. In [15] a maximum likelihood estimation (MLE) method has been proposed to solve the inverse problem of scatterometry. We have applied this method including the roughness parameter $\sigma_{\mathrm{r}}$ by an order dependent damping factor according to Eq. (7), i.e., the model function $\tilde{f_{n_{j}}}(\mathbf{p})$ is represented as $\tilde{f_{n_{j}}}(\mathbf{p})=e^{-\sigma_{\mathrm{r}}^{2}\left(\frac{2 \pi n_{j}}{d}\right)^{2}} \cdot f_{n_{j}}(\mathbf{p})$ in order to get more reliable estimations of the profile parameters and their uncertainties.

Under real experimental conditions efficiencies $y_{j}$ exhibit deviations from the model function $\tilde{f_{n_{j}}}(\mathbf{p})$ and are given by $y_{j}=\tilde{f_{n_{j}}}+\epsilon_{j}$. Here, $\mathbf{p}$ depicts the grating profile parameters like line widths, layer heights and side-wall angles and $\epsilon_{j} \sim \mathcal{N}\left(0, \sigma_{j}\right)$ describes a Gaussian noise with zero mean. The

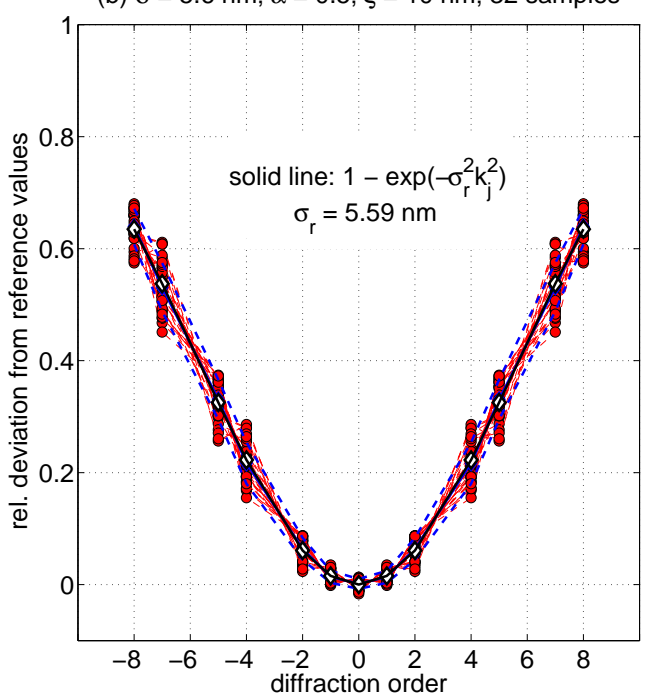

FIG. 8 Normalized deviations from the efficiencies of the unperturbed reference line structure, depicted as circles; diamond symbols represent the mean deviations of all samples; dashed lines indicate the mean efficiency \pm standard deviation; solid lines depict the exponential approximation and indicate symmetrical behaviour for both cases (a) and (b); (a) for FEM simulations and (b) for 2D-Fourier simulations of a $2 \mathrm{D}$-binary grating. 


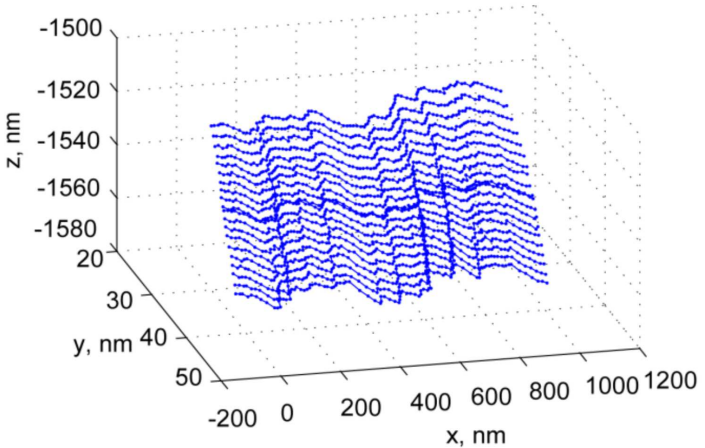

(a)

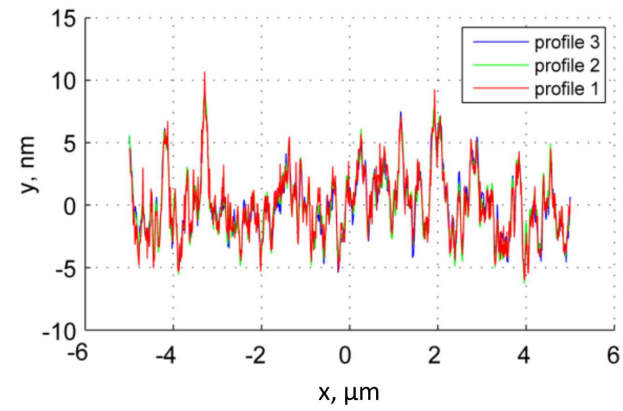

(b)

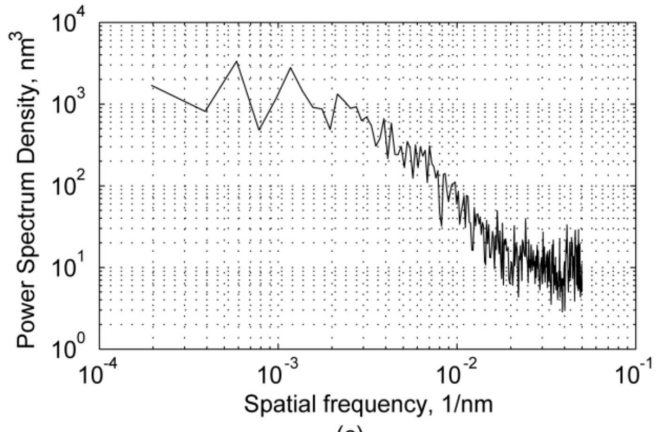

(c)

FIG. 9 Measurement of line edge roughness by the 3D AFM using a CDR70 AFM tip, shown as (a) LER profiles measured at different heights of the $1 D$ grating pattern of the die D8; (b) LER profiles measured at three different heights of an $1 \mathrm{D}$ grating pattern at the die $\mathrm{F}_{5}$; (c) calculated power spectrum density curve of the LER profile shown in (b).

variance $\sigma_{j}^{2}$ is chosen according to $\sigma_{j}^{2}=\left(a \cdot \tilde{f_{n_{j}}}(\mathbf{p})\right)^{2}+b^{2}$, in which $b$ is related to background noise and $a$ models power fluctuations of the incidental beam during the measurement process. Note that the model function $f_{n_{j}}(\mathbf{p})$ of a real photo mask, whose absorber lines are composed of several trapezoidal layers of different materials, has to be calculated rigorously, e.g. by FEM.

The model function together with the error model determine the following likelihood function

$$
\mathcal{L}\left(a, b, \mathbf{p}, \sigma_{\mathbf{r}}\right)=\prod_{j=1}^{m} \frac{1}{\sqrt{2 \pi} \sigma_{j}} \exp \left[-\frac{\left(\tilde{f_{j}}(\mathbf{p})-y_{j}\right)^{2}}{2 \sigma_{j}^{2}}\right],
$$

and its maximization yields the estimates of the desired parameters $\hat{\theta}=\left(\hat{a}, \hat{b}, \hat{\mathbf{p}}, \hat{\sigma}_{\mathrm{r}}\right)[15,16]$, i.e.

$$
\hat{\theta}=\arg \max \mathcal{L}\left(a, b, \mathbf{p}, \sigma_{\mathrm{r}}\right) .
$$

Uncertainties related to the estimates are given by the inverse of the Fisher-information matrix

$$
\mathbf{I}_{i j}=\left.\left(\frac{-\partial^{2} \log \mathcal{L}}{\partial \theta_{i} \partial \theta_{j}}\right)\right|_{\theta=\hat{\theta}},
$$

via $\Sigma_{i j}=\mathbf{I}_{i j}^{-1}$, that determines the variance $\operatorname{var}\left(\hat{\theta}_{i}\right)=\Sigma_{i i}$ of parameter $\hat{\theta}_{i}$.

The reconstructed line widths and the evaluated side-wall angles of experimental data, applying this MLE method (cf. also [23]), are in good agreement with measurements from 3D AFM of the same photo mask. The structural details of the measured EUV test photo mask are given in [5]. The applied
3D-AFM method and the CD comparison with scatterometry are presented in [24].

These results strongly supports the inclusion of line roughness by the parameter $\sigma_{\mathrm{r}}$ in form of the proposed damping factor (cf. Eq. (7)) and the simultaneous determination of $\sigma_{\mathrm{r}}$ along with the profile parameters $\mathbf{p}$. In Table 1 the reconstructed values for $\sigma_{\mathrm{r}}$ of the six different fields of the above-mentioned photo mask are given. Note that the investigated fields have different line-to-space ratios in the range of 0.5 to 3.0.

\subsection{Measurements of LER using AFM}

AFM technique has been widely applied in nanometrology. It allows direct and nearly non-destructive measurements of the 3D shape of nanostructures with both, a high lateral and vertical resolution. Compared to the scatterometry, it is less model and material dependent due to its relatively straight forward measurement principle. Therefore, we use EUV scatterometry as a potential high-throughput measurement method and AFM as a reference metrology tool.

A 3D AFM has been built up at PTB recently [25]. It is able to probe surfaces in arbitrary planes, e.g. $x z, y z$, and $x y$. This feature allows very convenient and direct measurements of sidewalls along the feature line. Some measurements of the $1 \mathrm{D}$ grating patterns of an EUV photo mask are demonstrated in Figure 9. All measurements were performed using a flared AFM tip CDR 70 (Team Nanotec $\mathrm{GmbH}$ ) having a tip radius of approximately $35 \mathrm{~nm}$. The first test measurement has been performed on the die D8, where forty LER profiles of a line 


\begin{tabular}{|c||cccc|cc|}
\hline $\begin{array}{c}\text { field } \\
\mathrm{L}: \mathrm{S} / \mathrm{nm}\end{array}$ & $\mathrm{D} 4$ & $\mathrm{D} 8$ & $\mathrm{~F} 6$ & $\mathrm{H} 8$ & $\mathrm{~F} 5$ & $\mathrm{G} 5$ \\
$540: 180$ & $540: 180$ & $540: 180$ & $540: 180$ & $180: 360$ & $160: 320$ \\
\hline \hline$\sigma_{\mathrm{r}} / \mathrm{nm}$ & 3.3 & 3.2 & 2.7 & 2.9 & $\mathbf{4 . 1}$ & $\mathbf{4 . 3}$ \\
\hline $\mathrm{std} / \mathrm{nm}$ & \pm 0.5 & \pm 0.4 & \pm 1.6 & \pm 1.2 & \pm 0.7 & \pm 0.9 \\
\hline \hline$\sigma_{\mathrm{RMS}} / \mathrm{nm}$ & & & & & $\mathbf{2 . 8}$ & $\mathbf{2 . 8}$ \\
\hline $\mathrm{std} / \mathrm{nm}$ & & & & & \pm 0.3 & \pm 0.3 \\
\hline \hline
\end{tabular}

TABLE 1 Values of reconstructed $\sigma_{\mathrm{r}}$ for six different mask fields; for die $\mathrm{F}_{5}$ and $\mathrm{G}_{5}$ the $\sigma$ values determined by $3 \mathrm{D}$ AFM are given additionally.

feature have been measured at different heights with a profile length of $1 \mu \mathrm{m}$ and a sampling distance of $10 \mathrm{~nm} /$ pixel, as shown in Figure 9(a). It can be seen that the profiles are similar, indicating that the sidewall topography has through variations from the top plane to the bottom plane. As the measured profile with a length of $1 \mu \mathrm{m}$ is too short to represent more lower spatial frequency components of LER, measurements with a profile length of $10 \mu \mathrm{m}$ and a sampling distance to $5 \mathrm{~nm} /$ pixel have been carried out on the die F5 and G5. A typical measurement result of the die F5 is shown in Figure 9(b), where three LER profiles measured at different heights are depicted. Again, these profiles look similar. Figure 9(c) depicts the power spectrum density curve calculated from the profiles shown in Figure 9(b).

However, it should be stressed that the performed AFM measurements are still quite preliminary yet. For instance, only the LER of two die structures have been investigated and the measurements are performed with only one AFM tip. The number of measurement repeats is very limited. Therefore, more investigations are to be carried out in the near future.

\subsection{Comparison of LER obtained by scatterometry and AFM}

Comparison of the measurement results between the PTB EUV scatterometer and 3D AFM are summarized in the Table 1. Structures of six dies with different line space ratios have been measured by the EUV scatterometer, while only two of them are measured by the 3D-AFM. In the table, the row of the data $\sigma_{\mathrm{r}}$ is obtained by reconstructing the measured scatterometric intensities using the extended model, as detailed in the Section 4.1. The row of the data $\sigma_{\mathrm{RMS}}$ is the RMS roughness value evaluated from the LER profiles as shown in Figure 9(b). No filtering has been applied in the data evaluation. The mean value and the standard deviation of results of 7 repeat measurements are given in the table.

As can be seen, the $\sigma_{\mathrm{RMS}}$ obtained by the $3 \mathrm{D}$-AFM is smaller than the $\sigma_{\mathrm{r}}$ obtained from the scatterometry. Relative to the scatterometric values the reduction is $32 \%$ for die F5 and $35 \%$ for die G5. Such deviation can be well explained by the principal difference of the two applied methods. From the viewpoint of signal processing, each measurement instrument can be regarded as a transfer function acting on the signal to be measured. The bandwidth with respect to spatial frequencies of the measurement function of the AFM instrument is limited in the used modes of measurement in this study. These constraints are determined by the applied measurement range along the sidewall edges and the ball shaped geometry of the used measurement tip. They provoke, that the roughness components with spatial frequencies lower than $0.2 \mu \mathrm{m}^{-1}$ and higher than $50 \mu \mathrm{m}^{-1}$, respectively, couldn't be measured by the applied AFM (cf. Figure 9(c)). While the scatterometer performs measurements over an area with a size of approximately $0.7 \mathrm{~mm} \times 0.7 \mathrm{~mm}$ with a radiation wavelength of 13.5 $\mathrm{nm}$, the spectrum of its measurement function was significantly wider than that of the applied AFM method, consequently leads to higher roughness results.

\section{DISCUSSION AND CONCLUSION}

We have proposed a 2D-Fourier transform method as a simple and efficient algorithm to complement previous investigations on the systematic impact of line edge roughness on light diffraction pattern of periodic line-space structures. This has particular been done in the EUV regime, where the light diffraction pattern is characterized by many significant wave modes with higher diffraction orders. The irradiance of illuminated rough apertures far away from the source plane is numerically calculated very efficiently as the 2D-Fourier transform of the light distribution in the aperture plane and then compared to those of the unperturbed, 'non-rough' aperture. Rough random boundaries are generated for the slits of the aperture by PSD functions ensuring realistic line edge profiles comparable to those of 3D AFM measurements along the sidewalls of the absorber lines. It has been shown, that for apertures of a typical size of $1 \mu \mathrm{m}$ in both directions and a pixel size of $0.1 \mathrm{~nm}$ the spatial averaging for each sample of a rough aperture is strong that just a few samples (e.g. ten) are sufficient to reveal a stable bias of the mean values at different diffraction orders. Compared to the diffraction pattern of the unperturbed aperture, the mean efficiencies of the rough apertures are revealing a systematic exponential decrease for higher diffraction orders. The standard deviation of the edge fluctuations $\sigma_{\mathrm{r}}$ and the diffraction order $n_{j}$ govern the revealed exponential damping factor. Ensembles of rough apertures with different values for the imposed standard deviation $\sigma$, the linear correlation length $\xi$, and the roughness exponent $\alpha$ have been investigated. Only a slight increase within a range of $5 \%$ has been found for the determined $\sigma_{\mathrm{r}}$ compared to the imposed $\sigma$ of the associated rough ensemble. Former results, obtained by rigorous computational expensive FEM computations have been confirmed on the base of a much more realistic model of line edge roughness.

The implicated model extension for scatterometry by a damping factor for the calculated efficiencies allows to determine the standard deviation $\sigma_{\mathrm{r}}$ of line edge roughness along with the critical dimensions of the periodic surface structure. The uncertainties of the determined values of $\sigma_{\mathrm{r}}$ are given as their standard deviations. They were estimated by the Fisher information matrix during the MLE approach to solve the inverse problem of scatterometry (cf. Eq. (10)). For six different dies of an EUV photo mask the $\sigma_{\mathrm{r}}$ values have been scatterometrically determined and lie in a range from $2.7 \pm 1.6 \mathrm{~nm}$ to $4.3 \pm 0.9 \mathrm{~nm}$. Note, that the measured EUV mask was fabricated in 2005 and that the quality of the production process for such photo masks has been improved by now. 
For two of the investigated dies of the photo mask, 3D AFM measurements have been performed too and yield to a $\sigma_{\mathrm{RMS}}$ value of $2.8 \pm 0.3 \mathrm{~nm}$ for both dies, where the given uncertainty is the standard deviation of repeated measurements. Due to the significantly smaller expansion of the AFM measurement region, roughness contributions from spatial frequencies below $0.2 \mu \mathrm{m}^{-1}$ could not be detected by AFM and the AFM results are smaller than those of the scatterometry. The comparisons presented are still preliminary results and further studies including more measured dies and further improved AFM measurement procedures need to be performed to further rate the quality of the agreement.

\section{ACKNOWLEDGEMENTS}

Results are part of the projects IND17 Scatterometry and NEW04 Uncertainty within the European Metrology Research Programme (EMRP).

\section{References}

[1] C. J. Raymond, M. R. Murnane, S. L. Prins, S. Sohail, H. Naqvi, J. R. McNeil, and J. W. Hosch, "Multiparameter grating metrology using optical scatterometry," J. Vac. Sci. Technol. B 15(2), 361-368 (1997).

[2] M.-A. Henn, R. Model, M. Bär, M. Wurm, B. Bodermann, A. Rathsfeld, and H. Gross, "On numerical reconstructions of lithographic masks in DUV scatterometry," Proc. SPIE 7390, 73900 Q (2009).

[3] J. Perlich, F. M. Kamm, J. Rau, F. Scholze, and G. Ulm, "Characterization of extreme ultraviolet masks by extreme ultraviolet scatterometry," J. Vac. Sci. Technol. B 22, 3059 (2004).

[4] F. Scholze, and C. Laubis, "Use of EUV scatterometry for the characterization of line profiles and line roughness on photomasks," in Proceedings to Mask and Lithography Conference, 1-9 (EMLC, Dresden, 2008).

[5] F. Scholze, C. Laubis, U. Dersch, J. Pomplun, S. Burger, and F. Schmidt, "The influence of line edge roughness and CD uniformity on EUV scatterometry for CD characterization of EUV masks," Proc. SPIE 6617, 66171A (2007).

[6] H. Gross, A. Rathsfeld, F. Scholze, and M. Bär, "Profile reconstruction in extreme ultraviolet (EUV) scatterometry: modeling and uncertainty estimates," Meas. Sci. Technol. 20, 105102 (2009).

[7] H. Patrick, T. Germer, R. Silver, and B. Bunday, "Developing an uncertainty analysis for optical scatterometry," Proc. SPIE 7272, $72720 \mathrm{~T}$ (2009).

[8] A. Kato, and F. Scholze, "Effect of line roughness on the diffraction intensities in angular resolved scatterometry," Appl. Optics 49(31), 6102-6110 (2010).

[9] H. Gross, M-A. Henn, S. Heidenreich, A. Rathsfeld, and M. Bär, "Modeling of line roughness and its impact on the diffraction intensities and the reconstructed critical dimensions in scatterometry," Appl. Optics 51, 7384-7394 (2012).
[10] B. C. Bergner, T. A. Germer, and T. J. Suleski, "Effective medium approximations for modeling optical reflectance from gratings with rough edges," J. Opt. Soc. Am. A 27, 1083-1090 (2010).

[11] C. A. Mack, "Analytic form for the power spectral density in one, two, and three dimensions," J. Micro-Nanolith. Mem. 10(4), 040501 (2011).

[12] C. A. Mack, "Generating random rough edges, surfaces, and volumes," Appl. Optics 52, 1472-1480 (2013).

[13] F. J. Torcal-Milla, L. M. Sanchez-Brea, and E. Bernabeu, "Diffraction of gratings with rough edges," Opt. Express 16, 19757-19769 (2008).

[14] T. Schuster, S. Rafler, V. F. Paz, F. Frenner, and W. Osten, "Fieldstitching with Kirchhoff-boundaries as a model based description for line edge roughness (LER) in scatterometry," Microelectron. Eng. 86, 1029-1032 (2009).

[15] M.-A. Henn, H. Gross, F. Scholze, M. Wurm, C. Elster, and M. Bär, "A maximum likelihood approach to the inverse problem of scatterometry," Opt. Express 20(12), 12771-12786 (2012).

[16] M-A. Henn, S. Heidenreich, H. Gross, A. Rathsfeld, F. Scholze, and M. Bär, "Improved grating reconstruction by determination of line roughness in extreme ultraviolet scatterometry," Opt. Lett. 37(24), 5229 (2012).

[17] J. W. Goodman, Introduction to Fourier Optics (Roberts at Company, Greenwood Village, 2005).

[18] 0. K. Ersoy, Diffraction, Fourier Optics, and Imaging (WileyInterscience, New York, 2006).

[19] D. Voelz, Computational Fourier Optics (SPIE Press, Bellingham, 2011).

[20] D. C. Champeney, Fourier transforms and their physical applications (Academic Press, London/New York, 1973).

[21] A. Tarantola, Inverse problem theory (Elsevier, Amsterdam, 1987).

[22] R.M. Al-Assaad, and D. M. Byrne, "Error analysis in inverse scatterometry I. Modeling," J. Opt. Soc. Am. A 24(2), 326-338 (2007).

[23] M-A. Henn, H. Gross, S. Heidenreich, F. Scholze, C. Elster, and M. Bär, "Improved reconstruction of Critical Dimensions in Extreme Ultraviolet Scatterometry by Modeling Systematic Errors," Meas. Sci. Technol., (2014), at press.

[24] G. Dai, K. Hahm, F. Scholze, M.-A. Henn, H. Gross, J. Fluegge, and H. Bosse, "Measurements of CD and sidewall profile of EUV photomask structures using CD-AFM and Tilting-AFM," Meas. Sci. Technol., (2014), at press.

[25] G. Dai, W. Hässler-Grohne, and D. Hüser, "New developments at Physikalisch-Technische Bundesanstalt in three-dimensional atomic force microscopy with tapping and torsion atomic force microscopy mode and vector approach probing strategy," J. MicroNanolith. Mem. 11(1), 011004 (2012). 